\title{
Defoliación y Remoción Parcial de la Panoja de Sorgo Durante la Etapa Post-Antesis y su Efecto en el Tamaño del Grano ${ }^{1}$
}

\author{
Raúl Rodríguez Herrera, Noé Montes García, Héctor Williams Alanis ${ }^{2}$
}

\section{COMPENDIO}

Con el objeto de determinar la importancia del área fotosintética y del tamaño de panoja en el peso específico del grano (PEG) de sorgo (Sorghum bicolor Moench), se llevaron a cabo dos experimentos: El primero consistió en eliminar en su totalidad las tres hojas superiores (S), medias (M) e inferiores (I) y las ombinaciones S+M, S+I, M+I, S+M+I además del testigo sin defoliar, en tres épocas (en antesis, 10 y 20 días después). En el sgundo experimento se eliminó la panoja en 0, 20, 40, 60 y 80 porciento, en tres épocas (en antesis, 10 y 20 días después).

Se desarrollaron los experimentos durante el ciclo primavera-verano 1989 en Río Bravo, Tamaulipas, en ambos se utilizó el híbrido RB-4000.

Los resultados indican que durante la noración las hojas que más contribuyen al PEG fueron las S y M, ya que si se eliminan estas hojas el PEG se reduce en un 11 y $15 \%$ con respecto al t,estigo, mientras que si se eliminan las (S) y (M), 10 ó 20 días de antesis el PEG se reduce en 25 y $2 \%$ respectivamente. Los tratamientos combinados $\mathrm{S}+\mathrm{M}, \mathrm{S}+1$ y $\mathrm{M}+\mathrm{I}$ durante la antesis y 10 días después, redujeron el PEG en mayor porcentaje que el tratamiento $\mathrm{S}+\mathrm{M}+\mathrm{I}$ fue el que más redujo el $\mathrm{PEG}$ en las tres épocas.

Por otra parte se observó que al eliminar un mayor porcentaje de la panoja el peso del grano se incrementa más, siendo mayor el incremento en la época de antesis seguida de 10 y 20 días después.

Palabras claves adicionales: peso de grano, floración.

\section{INTRODUCCIÓN}

El área agrícola del norte de Tamaulipas comprende un millón de hectáreas, de las cuales 720 mil se utilizan para . cultivar sorgo. dicha superficie produce dos millones de toneladas anuales de grano de sorgo, lo que convierte a la región en la principal área productora de este cultivo en el país.

Durante el desarrollo del cultivo se presentan una serie de eventos (plagas, enfermedades, granizados, etc.) los cuales 'reducen no solo el área laminar fotosintética sino que también a panoja de la planta dependiendo del estado de desarrollo de ésta y de la magnitud del evento.

El llenado de grano es una de las etapas en donde el daño por una plaga, enfermedad, etc. es más evidente ya que si este daño se realiza en la panoja, éste repercute directa-

\begin{abstract}
$^{3}$
Two experiments were conducted to determine the importance of photosynthetic leaf area and panicle size on final grain weight (FGW) in sorghum (Sorghum bicolor Moench). The first experiment consisted in defoliating the three upper (U), intermediate (M), and lower (L) leaves and different combinations $(\mathrm{U}+\mathrm{M}, \mathrm{U}+\mathrm{L}, \mathrm{M}+\mathrm{L}, \mathrm{U}+\mathrm{M}+\mathrm{L})$, plus a check, at three different times post-anthesis ( 0,10 and 20 days). The second experiment consisted in partially removing $0,20,40,60$, and $80 \%$ of the panicle at the same three times.

Experiments were conducted during the spring-summer season of 1989 in Rio Bravo, Tamnulipas, México, using hybrid RB-4000.

Results indicated that $\mathrm{U}$ and $\mathrm{M}$ leaves contributed the most to FGW at anthesis, as their defoliation reduced FGW by 11 and 15\% compared to the check. If upper or intermediate leaves were defoliated 10 or 20 days post-anthesis, FGW was reduced by $2 \mathrm{~S}$ and $2 \%$, respectively. Combined defoliation $(\mathrm{U}+\mathrm{M}, \mathrm{U}+\mathrm{M}, \mathrm{M}+\mathrm{L})$ during anthesis or 10 days arter reduced FGW more, with the treatment $\mathrm{U}+\mathrm{M}+\mathrm{L}$ having the greatest reduction in FGW at any time.
\end{abstract}

Removal of portions of the pnnicle increased FGW, the largest effect being detected during anthesis, followed by 10 and 20 days after.

mente en el rendimiento agronómico del cultivo, con base en lo anterior se realizó la presente investigación con el objeto de determinar el efecto de la defoliación post-antesis y de la remoción parcial de la panoja en el peso específico del grano de sorgo.

\section{REVISIÓN DE LITERATURA}

Ogunlela y Ologunde,(1983) al estudiar el efecto de la defoliación de las hojas en la etapa vegetativa y reproducti-

${ }^{1}$ Trabajo presentado en la XXXVI Reunión Anual del PCCMCA, San Salvador El Salvador C.A., marzo, 1990.

2 Investigadores de la Red de Sorgo y Mijo del CERIB. CIFAP- TAM., INIFAP. Apartado Postal 172. Cd. Río Bravo. Tam. México.

${ }^{3} \mathrm{El}$ abstract es traducción del compendio.

Publicado en Agronomía Mesoamericana, Vol. 2 (1991). 
va Del sorgo sobre la producción del grano en Nigeria consignan que la remoción de las hojas en la fase vegetativa reduce en una mayor Proporción el rendimiento del grano y las hojas superiores fueron más determinantes en la producción que las hojas y medias y las inferiores.

Por otra parte, Mendoza et al., (1985) mencionan después de realizar defoliaciones post-antesis en dos cultivares de sorgo en Zacatepec, Mor. México, que a medida que se incrementó el grado de defoliación, fue más drástica la reducción del rendimiento, asimismo la posición de las hojas defoliadas fue importante ya que la reducción en el rendimiento fue mayor cuando se defoliaron las hojas superiores.

La época de de foliación también juega un papel importante, dado que cuando se eliminan las hojas a la floración las reducciones en el rendimiento son mayores (70 \%), en comparación a cuando se realizan 20 días después, en esta época la reducción puede llegar hasta el 40 \% (Mendoza et al., 1986).

El rendimiento final de sorgo no solo está influenciado por la cantidad de área fotosintética presente en un cultivar, sino que también el tamaño de la panoja y el número de granos durante la etapa post-antesis juegan un papel importante, ya que constituyen la principal demanda de metabolitos durante dicha etapa (Ficher et al., 1976).

Sign y Colville, (1962) al realizar cortes del ápice y de la mitad de la panoja durante las etapas de emergencia de panoja y grano lechoso, con el objetivo de simular el daño de los pájaros, mencionan que la reducción en el rendimiento fue mayor cuando se realizó el corte en la etapa de grano lechoso y que la reducción del rendimiento fue mayor cuando se cortó la mitad de la panoja.

Por otra parte Khan et al., (1983) al remover 0, 10, 15 y 20 camillas primarias de la panoja en el estado de emergencia de la panoja de cinco cultivares de sorgo mencionan que existieron diferencias significativas entre cultivares para el rendimiento por planta, y que no existieron diferencias para esta característica entre los tratamientos de remoción de 0,10 y 15 ramillas.

\section{MATERIALES Y MÉTODOS}

La presente investigación se realizó en terrenos del Cmnpo Experimental del Instituto Nacional de Investigaciones Forestales y Agropecuarias (INIFAP) localizado en Río Bravo, Tam., el cual se encuentra situado a $25^{\circ} 5$ 9' latitud norte y $98^{\circ} 17^{\prime}$ de longitud oeste y a $30 \mathrm{msnm}$.

El trabajo consistió en dos experimentos, En el primero se sometió al cultivar RB-4000 a ocho niveles de de foliación (eliminación total de: las tres superiores (S), las tres medias (M) y las tres inferiores (I), las combinaciones
$(\mathrm{S})+(\mathrm{M}),(\mathrm{S})+(\mathrm{I}),(\mathrm{M})+(\mathrm{I}), \mathrm{S}+\mathrm{M}+\mathrm{I}$ además del testigo sin defoliar) en tres épocas: antesis, 10 y 20 días después. En el segundo experimento al mismo híbrido se le eliminó la panoja en 0 , 20, 40, 60 y 80 porciento de su tamaño, (estos cortes fueron realizados del ápice a la base empleando para ello tijeras podadoras) en tres épocas: antesis, 10 y 20 días después.

La fecha de siembra del cultivar utilizado fue del 8 de agosto de 1989, con sembradora mecánica, se aplicó un riego al día siguiente además se fertilizó con la fórmula 10000-00 al aporque y se realizaron dos escardas y se aplicaron dos riegos de auxilio.

Los tratamientos de ambos experimentos se distribuyeron bajo un diseño de bloques completos al azar con tres repeticiones, se empleó como unidad experimental cinco plantas con competencia completa. Después de cosechadas y trilladas las panojas, el grano fue pesado (sin glumas) y contados los granos por medio de un contador de semillas, con los datos de peso (P.G.) y número de granos (N.G.) se determinó el peso de 1000 granos (PEG) por medio de la fórmula:

$$
\mathrm{PEG}=\frac{\text { P. G. } \times 1000}{\text { N.G. }}
$$

En ambos experimentos se realizó un análisis de varianza individual para cada época, además de un análisis de varianza combinado (Tratamiento y época). En la comparación de las medias se utilizó la prueba de Tukey al $5 \%$.

\section{RESULTADOS Y DISCUSIÓN}

\section{a) Defoliación post-antesis}

A la floración se observaron diferencias significativas entre los tratamientos y entre las repeticiones, lo cual nos sugiere que la participación de los diversos estratos de hojas en el PEG es diferente (Cuadro 1) esto se puede corroborar con las pruebas de medidas (Cuadro 2). Cuando en esta etapa se defolió un solo estrato (S), (M), (I) el PEG se redujo en una menor proporción que cuando se defolió más de un estrato $(\mathrm{S}+\mathrm{M}),(\mathrm{M}+\mathrm{I}),(\mathrm{I}+\mathrm{M})$ y $(\mathrm{S}+\mathrm{M}+\mathrm{I})$; además, en esta etapa se aprecia que las hojas inferiores no tienen participación en el PEG ya que cuando se eliminaron el PEG fue similar al del testigo. Similares resultados fueron obtenidos por Mendoza et al., (1986) quienes mencionan que la posición de las hojas defoliadas fue importmlte y la importancia relativa de los estratos siguió el orden superior medias inferiores.

Diez días después de la antesis se encontraron diferencias altamente significativas entre tratamientos y entre repeticiones (Cuadro 1), lo que sugiere un nivel de participación diferente para cada estrato, en esta etapa se acentúa la importancia de las hojas superiores y en forma general se tiene un comportamiento similar a la etapa de floración ya que 
Cuadro 1 Significancia de las fuentes de variacón en los análisis individuales.

\begin{tabular}{lccc}
\hline \multirow{2}{*}{ Fuente de Variación } & \multicolumn{3}{c}{ Epoca } \\
\cline { 2 - 4 } & Floración & 10 Días & 20 Días \\
\hline $\begin{array}{l}\text { Defoliación } \\
\text { Nivel de defoliación }\end{array}$ & $*$ & $* *$ & - \\
Repeticiones & $*$ & $* *$ & - \\
CV & 13 & 12 & 9 \\
Remoción de Panoja & $* *$ & $* *$ & - \\
Porcentaje de corte & - & - & - \\
Repeticiones & 7 & 6 & 6 \\
CV & &
\end{tabular}

*,** Significativo al 5 y al $1 \%$ respectivamente, según prueba de $\mathrm{F}$

Cuadro 2 Efecto de los tratamiento de defoliación y remoción parcial de la panoja en post-antesis sobre el peso específico del grano.

\begin{tabular}{lllllll}
\hline Tratamiento & \multicolumn{5}{c}{ Epoca } \\
\cline { 2 - 6 } & Antésis & \multicolumn{3}{c}{ 10 Días después 20 Días después } \\
\hline Defoliación & & & & & & \\
Superior (S) & 19.2 & ac & 16.2 & bc & 21.1 & a \\
Medio (M) & 18.3 & ac & 19.9 & ab & 22.4 & a \\
Inferior (I) & 21.5 & a & 20.7 & ab & 23.6 & a \\
S+M & 15.9 & c & 17.1 & bc & 21.8 & a \\
S+I & 15.5 & c & 17.9 & ac & 23.4 & a \\
M+I & 17.2 & bc & 15.9 & c & 20.5 & a \\
S+M+I & 16.0 & c & 13.2 & d & 20.6 & a \\
Testigo & 21.5 & ab & 21.5 & a & 21.3 & a \\
& & & & & & \\
Remoción de Panoja & & & & & & \\
$0 \%$ & 21.5 & a & 22.8 & a & 23.1 & a \\
$20 \%$ & 23.8 & a & 24.3 & a & 23.9 & a \\
$40 \%$ & 30.0 & b & 29.5 & b & 24.2 & a \\
$60 \%$ & 31.5 & b & 29.9 & b & 24.5 & a \\
$80 \%$ & 33.8 & b & 35.7 & c & 21.5 & a \\
\hline
\end{tabular}

* Las medias con igual letra no son diferentes al $5 \%$ de probabilidad (Tukey).

el PEG se redujo más en la medida en que más estratos eran eliminados (Cuadro 2), estos resultados corroboran los obtenidos por Ogunlela y Ologunde (1983), quienes mencionan que a medida que se incrementa el grado de defoliación, fue más drástica la reducción en el rendimiento; similares resultados fueron obtenidos por Mendoza et al., (1986).

Para la fecha de 20 días después de la antesis no se encontraron diferencias significativas entre tratamientos ni entre repeticiones (Cuadro 1). Las medias de los diferentes tratamientos en esta época no fueron diferentes estadísticamen te, lo anterior nos sugiere que para esta época el PEG ya se definió y la contribución de las boj as a esta característicá es mínima en esta etapa en el RB-+OOO.
Cuadro 3 Significancia de las fuentes de variación en los análisis combinados.

\begin{tabular}{|c|c|c|c|c|}
\hline \multirow[b]{2}{*}{ Fuentes de Variación } & \multicolumn{4}{|c|}{ Experimentos } \\
\hline & & Defoliación & \multicolumn{2}{|c|}{ Remosión de Panoja } \\
\hline & $* *$ & \multicolumn{2}{|c|}{ ** } \\
\hline Tratamiento & & ** & \multicolumn{2}{|c|}{ ** } \\
\hline Epoca $\times$ Tratamiento & & -- & \multicolumn{2}{|c|}{ ** } \\
\hline $\mathrm{CV}$ & & 11 & \multicolumn{2}{|c|}{6} \\
\hline \multicolumn{5}{|c|}{${ }^{*},{ }^{* *}$ Significativo al 5 y $1 \%$ respectivamente según prueba de $\mathrm{F}$. } \\
\hline \multicolumn{5}{|c|}{$\begin{array}{l}\text { Cuadro } 4 \text { Porcentaje de reducción en el peso específico del grano por los } \\
\text { diferentes niveles de defoliación. }\end{array}$} \\
\hline \multirow{2}{*}{$\begin{array}{l}\text { Hojas } \\
\text { Eliminadas }\end{array}$} & \multicolumn{4}{|c|}{ Días después de la antesis } \\
\hline & 0 & & 10 & 20 \\
\hline Superiores (S) & 11 & & 25 & 1 \\
\hline Medias (M) & 15 & & 7 & +5 \\
\hline Inferiores (I) & 0 & & 4 & +11 \\
\hline $\mathrm{S}+\mathrm{M}$ & 26 & & 20 & +2 \\
\hline$S+I$ & 27 & & 17 & +10 \\
\hline$M+I$ & 20 & & 26 & 4 \\
\hline $\mathrm{S}+\mathrm{M}+\mathrm{I}$ & 26 & & 39 & 3. \\
\hline
\end{tabular}

En el análisis combinado solo se detectaron diferencias significativas entre las épocas y entre los tratamientos, no así para la interacción, esto sugiere que la importancia relativa de cada estrato en cada etapa fue la misma (Cuadro 3 ).

Una mejor perspectiva del efecto del nivel de de foliación sobre la reducción del peso específico del grano (PEG) se aprecia en el Cuadro 4, en donde se puede observar que durante la floración y la etapa de 10 días después de antesis los estratos que más contribuyeron al PEG fueron los dos tercios superiores concordando con los resultados consignados por Mendoza et al., (1986), para un sorgo tolerante al frío. al respecto Srinivasa y Sing (1982) mencionan que los defoliación incrementa la eficiencia fotosintética de las bojas que permanecen y la eficiencia decrece grandemente de las bojas superiores a las inferiores.

En la etapa de 20 días después de la antesis se apreció que las hojas medias e inferiores no sólo no contribuyeron sino que además compitieron con el PEG por los fotosintatos. Al respecto Mendoza et al., (1986) indican que la panoja del tallo principal no fue la única de fotosintetizados durante la etapa de post-antesis en un sorgo tolerante al frío.

\section{b) Remoción parcial de la panoja}

En el ANVA tanto en floración como a diez días después de la antesis se encontraron diferencias significativas entre 
Cuadro 5 Porcentaje de Reducción del Peso Específico del grano por los diferentes cortes de la panoja en tres épocas.

\begin{tabular}{cccc}
\hline \multirow{2}{*}{$\begin{array}{c}\text { Porcentaje de } \\
\text { Remoción }\end{array}$} & \multicolumn{3}{c}{ Días después de la antesis } \\
\cline { 2 - 4 } & 0 & 10 & 20 \\
\hline 0 & 0 & 0 & 0 \\
40 & 11 & 15 & 17 \\
60 & 16 & 22 & 37 \\
80 & 41 & 48 & 58 \\
\hline
\end{tabular}

tratamientos (Cuadro 1). En la etapa de floración así como diez días después de ésta, el grano tiende a crecer más a medida que se incrementa el porcentaje de corte (Cuadro 2), por otra parte no se encontraron diferencias significativas entre el nivel de corte del $20 \%$ y del testigo $(0 \%)$, similares resultados fueron obtenidos por Sing y Colville, (1962) cuando eliminaron el ápice de la panoja y por Mishra y Dabholkar, (1983) al eliminar 10 y 15 ramillas primarias.

Al no haber diferencias significativas entre los tratamientos en la etapa de 20 días después de la antesis se confirma que el PEG ya se definió para esta etapa ya que era de esperarse que al haber menor demanda (tamaño de panoja) pero conservando las plantas la misma área foliar fotosintética, los granos de la panoja tendieron a crecer más; sin embargo, lo anterior no se aprecia.

Al realizar el análisis combinado se detectaron diferencias significativas entre las fuentes de variación, épocas, tratamientos y la interacción época $\mathrm{x}$ tratamiento. Con respecto a épocas se observa que en la etapa de 20 días el grano que permanece en las panojas después de los cortes, tiende a crecer menos, esto es; que si en esta etapa el sorgo sufre un dafio a la panoja: v.g. ataque de pájaros y estos consumen un determinado porcentaje de la panoja, la planta ya no compensa (con un mayor peso específico del grano) parte del dafio causado por las aves.

Una mejor comprensión del efecto del porcentaje de corte de la panoja sobre el porcentaje de reducción en peso específico del grano (PEG) se aprecia en el Cuadro 5. En donde se observa que a medida que el corte de la panoja se hace en una época más tardía durante el llenado del grano, la compensación de la planta con un mayor peso específico del grano es menor.

\section{CONCLUSIONES}

1. La participación de los estratos foliares es diferente en el PEG y la importancia relativa sigue el orden: superior medias inferiores en todas la épocas.

2. El PEG se reduce más a medida que se incrementa el número de estratos desfoliados.

3. En la etapa de 20 días después de la antesis las hojas ya no contribuyen a el PEG.

4. PEG aumenta en la medida en que aumenta el porcentaje de corte a la panoja.

5. En la etapa de 20 días después de la antesis el PEG ya se definió en el híbrido RB-4000.

6. La compensación de la planta con un mayor PEG es menor a medida que el corte se hace en una época más avanzada del llenado de grano.

\section{BIBLIOGRAFIA}

FICHER. K, S.; WILSON.G.L; DURTHIE.I. 1976. Studies of production in Sorghum bicolor (E. Moench). VII. Contribution of plant parts to canopy photosynthesis and grain yield in field stations. Aust. J. Agric. Res. 27: 235-242.

KHAN. R. V.; MISHRA, R.C.; DABHOLKAR, A.R, 1983. Effect of removal of primary branches on grain yield. Sorghum Newsletters. 132-133.

MENDOZA O. L.E.; CORONA T., T.; AVELLANA B.,J. 1985. Defoliación post-antesis, rendimiento de grano y sus componentes en tallos principales de dos cultivares de sorgo. Agrociencia 62: 87-99.

MENDOZA O., L.F.; CASTELLANOS S.,A.; LIVERA M..M. 1986. Defoliación a partir de antesis. rendimiento de grano y distribuci6n final de materia seca de un sorgo (Sorghum bicolor (L. Moench) tolerante al frío. Agrociencia 65: 253262.

OGUNLELA, V. B.; OLOGUNDE, O.O. 1983. Response of sorghum yield and yield components to partial defoliation. Sorghum Newsletters. 127-128.

SINGH. S. S.; Colville,W.L. 1962. Effect of clipping on yield and certain agronómic characters of irrigated grain sorghum. Agronomy Journa1 54: 484-486.

SRINIVASA R.. N. K. y S. P. SINGH. 1982. DefQliation andcompensatory mechanism in sorghum. Indian J. Agric. Sci. 52 (11): 748-75. 